Jahír Alexánder Gutiérrez Ossa*

Gleidy Alexandra Urrego Estrada*"

Jefferson Carmona Maldonado

Institución Universitaria Tecnológico de Antioquia, Medellín, Colombia

Recibido: 22 de octubre de 2015

Concepto de evaluación: 16 de mayo de 2016

Aprobado: 27 de mayo de 2016

Artículo de investigación (C) 2016 Universidad Católica de Colombia. Facultad de Ciencias Económicas y Administrativas. Todos los derechos reservados

* Economista; PhD. en Administración Pública; Magíster en Desarrollo. Estudios en Especialización de Sistemas de Información Geográfica. Dirección de correspondencia: Calle 72A N $73-45$ apto 306, Pilarica

Blue, Medellín, Antioquia. Correo electrónico: jagogutierrez@gmail.com

** Administradora Pública; Magíster en Hábitat. Doctorando en Ciencias Humanas y Sociales, Universidad Nacional de Colombia. Miembro del grupo de investigación Observatorio Público, de la Institución Universitaria Tecnológico de Antioquia. Dirección de correspondencia: Calle $48 \mathrm{~N}^{\circ}$ 35-19 apt 302, Barrio Buenos Aires, Medellín, Antioquia. Correo electrónico: gleidy.urrego@gmail.com
Finanz. polit. econ., ISSN: 2248-6046, Vol. 8, No. 2, julio-diciembre, 2016, pp. 251-269 http://dx.doi.org/10.14718/revfinanzpolitecon.2016.8.2.3

\section{Políticas públicas desde las crisis económicas y sociales, en el marco de los Estados y territorios emergentes*}

\section{RESUMEN}

En el presente artículo se aborda la política pública en dos aspectos: por una parte, la emergencia social, la crisis institucional y económica en algunos países de Latinoamérica, así como su estructura compleja y sistémica; por otra, la formulación e implementación de las políticas públicas, que son consideradas en el marco de las crisis internacionales de los estados de emergencia. Por medio del análisis multicriterio se establecerán los argumentos relacionados con la capacidad de gestión, los resultados o el trabajo en diferentes sectores. Luego, mediante el designing process, se sustentará explicativamente el problema a través de la revisión económica, jurídica, política y social. Finalmente, se valoran las bondades de las políticas públicas en las situaciones trascendentales o difíciles que requieran de su asomo o su plena presencia.

Palabras clave: administración pública, crisis internacional, política pública, externalidades, modelos de planificación.

JEL: H720, H750, H120, J1

\section{Public policies from economic and social crisis in the framework of emerging States and territories}

\section{ABSTRACT}

The present article approaches two aspects of public policy. On the one hand, we have social emergence, the institutional and economic crisis in some Latin American countries, as well as its complex and systematic structure. On the other, it looks at the formulation and implementation of public policies, considered within the framework of the international crises of

\footnotetext{
* Este artículo es producto del proyecto de investigación Evaluación del Club de las Buenas Prácticas de la OCDE en la relación Estado-empresa en Colombia: hacia la gestión empresarial público-privada internacional a través de la formulación de políticas públicas, realizado por el Grupo de Investigación Observatorio Público, de la Institución Universitaria Tecnológico de Antioquia, entre abril de 2015 y mayo de 2016.
} 
*** Administrador Público. Estudios en Políticas Públicas con Enfoque en Derechos Humanos. Miembro del grupo de investigación Observatorio Público, de la

Institución Universitaria Tecnológico de Antioquia. Docente de cátedra en la Institución Universitaria Tecnológico de Antioquia y la Institución Universitaria Esumer, Medellín, Colombia. Dirección de correspondencia: Calle $116 \mathrm{~N}^{\circ}$ 64D-18 piso 2 . Barrio Boyacá las Brisas, Medellín, Antioquia. Correo electrónico: jefersoncm8@gmail.com the states of emergency. Through a multi-criteria analysis, arguments related to the management capacity, and the results or work in different sectors are established. Then, using a process of design, the problem will be explained through a review of the economic, legal, political, and social arenas. Finally, the virtues of the public policies in transcendental or difficult situations that require their appearance or full presence will be evaluated.

Keywords: public administration, international crisis, public policy, externalities, planning models, public policies.

\section{Políticas públicas a partir das crises econômicas e sociais no âmbito dos Estados e territórios emergentes}

\section{RESUMO}

Neste artigo, aborda-se a política pública em dois aspectos: por um lado, a emergência social, a crise institucional e econômica em alguns países da América Latina bem como sua estrutura complexa e sistêmica; por outro, a formulação e implantação das políticas públicas que são consideradas no âmbito das crises internacionais dos estados emergentes. Por meio da análise multicritério, serão estabelecidos os argumentos relacionados com a capacidade de gestão, os resultados ou o trabalho em diferentes setores. Em seguida, mediante o designing process, será sustentado, de forma explicativa, o problema por meio da revisão econômica, jurídica, política e social. Finalmente, avaliamse os benefícios das políticas públicas nas situações transcendentais ou difíceis que requerem de seu aparecimento ou sua plena presença.

Palavras-chave: administração pública, crise internacional, política pública, externalidades, modelos de planejamento. 


\section{INTRODUCCIÓN}

El alcance de la política pública corresponde a un objetivo político de naturaleza democrática que involucra la participación de diferentes actores y propende a la consecución de resultados (Lahera, 2004). Desde luego, la política pública articula un deber ser en cuanto a desarrollar capacidad institucional, civil y económica, que se ofrece como fundamento para la aplicación o generación de propuestas de índole cultural, económica, social y política. El papel de orientador y medidor de las acciones realizadas en dicho frente ha ganado relevancia en los últimos años. En este sentido, la política pública ha logrado establecer una especie de mampara a la hora de establecer los impactos y resultados esperados de actividades emergidas o complementarias desde la promoción de políticas en general.

Desde este referente, el presente artículo aborda la política pública en clave del enfoque de gobernabilidad, que supone la capacidad que necesariamente deben tener los gobiernos para producir acciones, potencialidades legales, fiscales, informativas, políticas y gerenciales, así como resultados enfocados a resolver problemáticas públicas (Aguilar, 2007). El camino tomado por la política pública, dirigido hacia el cuestionamiento y la interrogación sobre las acciones ejecutadas en los países latinoamericanos, hace eco a la hora de evaluar las instituciones del orden gubernamental. Igualmente, sirve para incluir en la propia gestión estatal elementos no tenidos en cuenta, los cuales, finalmente, provocan en la política propuesta una revisión plena de hasta dónde se podrían comprometer o vincular diferentes intereses. Es el marco de convergencia, más que el de la crítica, el que paralelamente le ha dado objetividad.

Por otra parte, las políticas públicas, especialmente en América Latina, sufren un desgaste social y político debido a la crisis del estado benefactor, que las hace ineficientes y a favor de procesos electorales (Bayce, 2005). Sin embargo, es la política pública, desde los gobiernos, la que permite un enfoque y una continuidad del objetivo colectivamente determinado para afrontar las crisis sociales, políticas y económicas (Feoli, 2011). Por ende, la política pública pasa de ser el instrumento dedicado a la evaluación de la intervención del Estado y de los programas a cargo de diferentes estamentos gubernamentales, para instaurarse ahora como el espacio en el que se generen condiciones y propuestas que puedan contribuir a mejorar las condiciones en que se presentan las actividades que convocan a la población y los territorios. Desde dicha dirección, la política pública en América Latina no solo ha invitado a la cohesión de cada país, sino que ha llamado a la reflexión internacional con respecto a su potencial, a partir del cual ampliamente puede considerarse el marco intergubernamental que arrastra.

La pérdida de gobernabilidad económica de los estados en América Latina se profundiza en un clima político partidista y mediático; allí necesariamente deben plantearse alternativas sistémicas y no coyunturales en los planos social, climático, energético y alimentario. El carácter dimensional logrado por la política pública contrasta con el afán de procurar, desde ella, un espacio de fundamentación académica y teórica (Dowbor, 2009). Precisamente, la capacidad de incubación multifacética permite establecer el grado de rigurosidad que puede alcanzar la política pública en cualquier escenario. De ahí que el tamizaje al cual se somete la política pretenda precisamente la distribución de ingresos, oportunidades y capacidades a la mayoría de la ciudadanía (Mendíaz, 2004), más aún, en situaciones tan concretas y dispares como la actual crisis internacional, que, de manera reiterada, pone en duda la capacidad de las acciones procuradas por los gobiernos y las instituciones para mitigar o superar sus consecuencias.

La intervención que compromete la política pública parte de una correlación más compleja que dé cuenta de un contexto específico (Manuel, 2005); esto es, la política pública que, para el interés del este artículo, está contenida en la intervención de las crisis económicas y sociales 
que se suscriben a territorios democráticamente emergentes. Así, la política pública contextualizada en el ámbito territorial facilita los problemas del poder y la negociación (Cardozo, 1990) entre diferentes actores sociales, políticos y económicos, con ventajas que van desde lo colectivo (bienestar social) y lo ambiental, hasta robustecer el espíritu democrático (Elizalde, 2003).

Frente a las crisis que afrontan las políticas públicas, el papel de los Estados latinoamericanos se focaliza en una escala local y otra global, en función de generar equilibrios internos (Dowbor, 2009) para la restauración de lo público (Sojo, 2005) y establecer las bases de dirección y fundamentación del Estado (Giménez, 2011). Desde luego, el territorio, como contendor material y construcción social en la política pública, está cuantificado por el desarrollo y la capacidad económica que deben ser promocionados desde una gestión local (Lira, 2003). Las crisis económicas y sociales, en el marco de los Estados y territorios emergentes y en relación con las políticas públicas, demandan capacidad de gobierno en temas de déficit público (García, 2007) y transformaciones burocráticas del Estado (Varela, 2005).

La ausencia de visión sistémica en las políticas públicas (Babino, 2009) en países latinoamericanos para afrontar las crisis económicas y sociales recae en la preocupación de los gobiernos, sus administraciones y su acción pluralista (Tamayo, 1997). Además, la visión sistémica implica a su vez la articulación con otras políticas públicas (Arroyave, 2010) que permitan una intervención legítima y global en este contexto. El problema que aborda este artículo de investigación es establecer el papel que puede desempeñar la política pública en medio de la crisis institucional, en materia económica y política; además, analiza las consecuencias culturales y sociales que hoy se generan en los países de América Latina, con el fin de orientar y beneficiar a los diseñadores de las políticas públicas y a los que acuden a ellas en busca de soluciones, lo que implica mejorar la interacción y la sinergia de las diversas propuestas que de allí emanan.
Este artículo tendrá como marco de referencia los siguientes puntos: en primer lugar, examinar la política pública frente a la emergencia de la sociedad latinoamericana y la crisis institucional del estado internacional; en segunda instancia, dilucidar la estructura compleja y sistémica de la política pública ante la crisis y la emergencia internacional en los países latinoamericanos emergentes; finalmente, identificar la crisis internacional y los estados de emergencia entre estos países desde las políticas públicas, con una aproximación específica a algunos de ellos.

\section{METODOLOGÍA}

Para la consecución de los resultados que se plantean en este artículo, se partió de un método deductivo cuya herramienta de recolección de información fue la lectura de textos complementarios, en un marco de los Estados emergentes; además, se hizo la revisión bibliográfica de diversos autores frente al Estado, las crisis económicas y sociales y su relación con las políticas públicas.

La metodología de investigación se apalanca en dos instrumentos fundamentales para la elaboración del contenido marco: en primer lugar, el análisis multicriterio, cuya base de trabajo es la utilización de instrumentos teóricos para establecer los argumentos que permiten aducir sobre la capacidad de gestión ciertos resultados o trabajo en diferentes sectores; en segundo lugar, el designing process, que consiste en la sustentación explicativa del problema u objeto de estudio, a través de la revisión económica, jurídica, política y social del tema.

El primer ejercicio metodológico se caracteriza por ser de corte más argumentativo, en tanto el segundo permite la sustentación del análisis desde diferentes fuentes del conocimiento, bajo una sola estructura. Ambos ejercicios metodológicos son abordados en la aproximación bibliográfica de algunos países latinoamericanos considerados como Estados y territorios emergentes. 


\section{RESULTADOS}

Las políticas públicas son intenciones de gobierno con la finalidad de organizar, administrar y controlar las acciones del Estado y sus intereses. Parten de una idea generalizada: la transformación social por medio de la inclusión y la participación. Además, están encaminadas a ofrecer soluciones a las emergencias de carácter institucional, en los aspectos económicos, políticos y sociales, así como las derivadas del contexto internacional. Dicha acción debe tener un carácter de excelencia o, como mínimo, propositivo e intencional:

Una política pública de excelencia corresponde a aquellos cursos de acción y flujos de información relacionados con un objetivo político definido en forma democrática; los que son desarrollados por el sector público $y$, frecuentemente, con la participación de la comunidad y el sector privado. Una política pública de calidad incluirá orientaciones o contenidos, instrumentos o mecanismos, definiciones o modificaciones institucionales, y la previsión de sus resultados (Lahera, 2004, p. 8).

En otras palabras, las políticas públicas son pactos entre el Estado y la sociedad que buscan no solo la solución de problemas como medida de choque, sino también la consensuada planeación para la prevención de dichas dificultades. En ese orden de ideas, se abordan tres puntos esenciales para identificar el problema central del artículo: en primer lugar, se efectúa un análisis de los elementos y las circunstancias que rodean la política pública en momentos de crisis y oportunidad; en segunda instancia, la revisión de esas crisis institucionales desde la perspectiva compleja y sistémica; finalmente, se visualiza la capacidad de respuesta que tienen los países emergentes frente a los desarrollados para responder a la crisis a través de las políticas públicas.

\section{Política pública frente a la emergencia de la sociedad latinoamericana y la crisis institucional del estado internacional}

La apertura global hacia diversos propósitos, amparada en la democratización planetaria, ha traído como marco de referencia paralelo una serie de problemáticas que aún en la actualidad corresponden a dificultades relacionadas con las condiciones estatales e institucionales de cada país. En otras palabras, la posición que se tiene respecto a los elementos que han provocado el estado de emergencia latinoamericano, sobre el que confluyen dificultades ambientales, laborales y planetarias, obedece estrictamente a la concepción de estructura que cada país ha elaborado para responder a las libertades internacionales:

Dos son las críticas principales al enfoque de la gobernabilidad, que nos es el más familiar. La primera crítica observa que es un enfoque centrado en las capacidades del gobierno, las cuales no son más que potencialidades de acción, y no en las acciones de relación del gobierno con la sociedad, que son las que hacen reales, manifiestas, efectivas las capacidades: las "actualizan" (en lenguaje aristotélico). La capacidad de gobernar se muestra y valida en los resultados reales que producen las acciones que ejecuta el gobierno. En los hechos, el gobierno decide y practica acciones que explotan a cabalidad las potencialidades legales, fiscales, informativas, políticas, gerenciales que posee, acreditando entonces que posee la capacidad de atender problemas y enfrentar desafíos públicos, pero también puede decidir y practicar acciones que las desaprovechan o subutilizan, con el resultado de agravar problemas y no impactar en las condiciones sociales indeseadas, transformándolas. En suma, las acciones (tipo de) que el gobierno practica en 
su interacción con los actores sociales para fines de dirección y coordinación son más decisivas que las capacidades que posee, en tanto las acciones son las que convierten la capacidad directiva en eficacia directiva. Dicho de otro modo, más determinante que la capacidad de gobernar es el proceso de gobernar con sus prácticas de interlocución y negociación con los actores sociales (cosa que la gobernanza resalta) (Aguilar, 2007, p. 5).

El proceso reduccionista al que ha sido confinado el Estado y el consecuente encumbramiento de las disposiciones económicas - elemento guía de los diversos flujos poblacionales y de movilidad de recursos - sustentan las bases para que los Estados comprendan la manera de ubicarse ante las oportunidades, pero igualmente de cara a las problemáticas, en un mundo que, en medio de la pretendida homogeneidad, no es capaz de responder de manera unilateral o por lo menos concomitante a problemáticas comunes. El desprestigio de los Estados latinoamericanos, llenos de agendas, pero carentes de políticas y ejecución, reviste hoy el carácter melancólico en el que se extiende la política internacional, que trata de buscar refugio en la política pública como diáspora situacional. Bayce (2005) argumenta en este sentido:

Sin embargo, a pesar de la crisis del Estado Benefactor, del desgaste de las políticas sociales públicas, autorreferidas, inerciales y distantes del mundo de la vida, de su insuficiente éxito profundizador, unido a su decreciente utilidad marginal en una posmoderna sociedad de consumo, esas políticas públicas sociales continúan siendo aplicadas $y$, aparentemente, con buen retorno político-electoral (p. 75).

La falta de oportunidades, catalogadas al paso de cada grupo o sujeto social, genera un salto para enfrentar por cuenta propia lo que para muchos no necesariamente cabe en el andamiaje de crisis; ellos aún dan por descontado como escenario de explicación al conflicto armado la desocupación laboral, la migración ilegal, la pobreza o la trata de personas. Este constituye el esquema de diálogo internacional a partir del cual se pretende ubicar las políticas públicas de manera programática, pero con pleno interés en que terminen por ser difundidas en los territorios en los que claramente se refleje el impacto. Así, la política pública ostenta el pliego de oportunidades que puede brindarse en medio de la crisis:

Como garantes del bienestar colectivo, los gobiernos deben intervenir ante choques externos, tanto para minimizar los impactos de la crisis como para facilitar la recuperación y sentar las bases para un crecimiento sostenido en el largo plazo. Como mínimo, las políticas públicas deben dirigirse a evitar los efectos más severos de las crisis sobre el empleo y los niveles de pobreza, previniendo que una crisis económica se convierta en una crisis social y política. Sin embargo, las políticas también deben ser estables y mantenerse enfocadas en el largo plazo, preservando un clima adecuado para la inversión y la innovación. (Landes, 1998) (Feoli, 2011, p. 8).

El bajo reconocimiento y sustento que tienen dichos elementos corre por cuenta de la poca asociación que existe a la hora de plantear soluciones tradicionales, con la posibilidad de incorporar elementos de la política pública, en orden a procurar el restablecimiento del orden institucional, enfrentar los elementos de crisis, solventar, con el paso de las propuestas, los impactos que no dan espera por cuenta de los colapsos y las incertidumbres que se presentan en la actualidad. En este escenario, claramente está en serio riesgo la capacidad de los Estados latinoamericanos para responder a sus ciudadanos, debido al complejo entorno derivado de la situación. La capacidad de respuesta del frente institucional es la que pone a prueba la implementación de la política pública, la cual 
responde con más vehemencia si es corroborada estamentalmente:

El caos sistémico y la clara pérdida de gobernabilidad económica, en un contexto de desesperación de una inmensa masa de personas perjudicadas, están generando un nuevo clima político. Se están abriendo nuevas posibilidades para colocar sobre la mesa propuestas más amplias, en el sentido de un desarrollo con pies y cabeza. Más precisamente, se abre un espacio para alternativas de desarrollo y para que - hoy ya no parece un objetivo imposible - nuestro propio dinero sirva para fines útiles. No se debe soñar en exceso, ya que buena parte del espacio político que se genere dependerá de la profundidad que alcance la crisis. Pero sí es importante plantear alternativas sistémicas, pues lo que estamos sufriendo es una crisis estructural de corto y mediano plazo dentro de un cuadro de crisis más amplias que se avecinan, particularmente en los planos social, climático, energético y alimentario, entre otros (Dowbor, 2009, p. 196).

Los elementos consecuentes de la actual crisis internacional plantean la necesidad de considerar aspectos sobre los que se debe trabajar para enfrentar lo que puede catalogarse otra crisis mundial; esta, aunque es semejante a la década de los treinta del siglo $\mathrm{XX}$, cuenta con más tecnología, pero con un mayor nivel de incertidumbre y desconfianza, a pesar de la abultada información que se dispone. La ausencia de bases y elementos para que la crisis sea sorteada recae de nuevo en la suerte que al respecto pueda ofrecer la política pública en los estamentos de orden estatal o no estatal, que tendrán como misión evaluar y orientar los requerimientos sobrevinientes por el desbarajuste en el modelo tradicional, contexto en el que las variables y los desajustes terminaban en equilibrio general:
El Estado debe construir nuevas políticas públicas que se constituyan como ámbitos de mediación entre la sociedad $y$ el mercado. Mediación que debe ser entendida como distribución de ingresos, oportunidades y capacidades a la mayoría de la ciudadanía. Realidades, "concretos históricos" que los enfoques neoinstitucionalistas no parecieran, de acuerdo a nuestro criterio, tener en cuenta al momento del análisis (Mendíaz, 2004, p. 36).

Es evidente que la marcada explicación apoyada en el reservorio de modelos aglutinados de datos, variables y correlaciones ha pasado de la asertividad a la incertidumbre, y de estas a la incapacidad de explicar lo que está sucediendo en el mundo. Esta incapacidad, de paso, se esta llevando por delante los logros obtenidos en materia de crecimiento, desarrollo y protección social, los cuales constituían el baluarte de la relación entre las medidas planteadas en materia de política estatal o institucional. Los resultados que de manera directa habrían de aparecer, terminaron trastocados por las quejas a los ingredientes e hicieron tambalear los alcances apreciados:

No puede desconocerse que el interés por el estudio de las instituciones se refleja en el aumento de los tratados sobre política, legislatura, gobierno local, políticas públicas, economía política, élites y culturas políticas ya no sólo a nivel de los Estados Unidos y Europa, sino en el contexto latinoamericano. Existen, por consiguiente, dentro del debate académico acerca de la revalorización del Estado y sus instituciones (Mendíaz, 2004, p. 29).

La explicación ha pasado de doctrina a modelo, y de estos a propuestas replicables que solo han servido para deteriorar los fundamentos desde los que se establecía el marco de dirección de política estatal en diferentes frentes. Ahora, comprometidos decididamente con políticas compromisarias de organismos multilaterales, 
los Estados en Latinoamérica no ven otro camino que el de auspiciar de manera discrecional y discreta ciertas políticas que han tratado de materializar en la fundamentación de las políticas públicas; sin embargo, los Estados no han comprendido uno de los principios de estas, a saber: deben ser parte de las pretensiones institucionales, y no consecuencia de las malas ejecuciones, criterio último que les resta validez. Manuel (2005) sostiene al respecto:

La relación intervención-políticas públicas muchas veces es planteada como "mecánica", donde la relación sujetopolíticas públicas se construye de manera automática, con muy pocas mediaciones, en general prestablecidas y en forma programada. Desde una mirada centrada en la intervención esta correlación se hace mucho más compleja y da cuenta de las posibilidades de dirección de éstas desde el terreno de la práctica. En otras palabras, la intervención le puede conferir singularidad a la aplicación de las políticas públicas, justamente en un contexto donde se ve la necesidad de transversalidad e integración de las mismas (p. 8).

El desánimo con la orientación dada a la política internacional por cuenta de los países latinoamericanos es consecuente con el descenso en su estabilidad. Particularmente en los países desarrollados, el agotamiento de las políticas estatales se ha venido evidenciando con el deterioro de las propuestas de política pública internacional que ellos mismos habían establecido como apoyo y sustento a la crisis de oportunidades, ejemplificadas en temas como la promoción a la migración internacional, la cooperación internacional o el acompañamiento a diferentes estamentos en múltiples tareas que, con cargo directo a una especie de misión internacional, eran dispuestas por países ricos a favor de los emergentes. Ahora la hoja de ruta cambia: quienes deben recibir ayuda son los países ricos, dadas las consecuencias nefastas que traerían en los emergentes:

Ésta es una etapa metodológicamente difícil, pero en la que existe una gran riqueza de propuestas en la que se conjugan disciplinas muy diversas. Podemos encontrar análisis predominantemente políticos para definir una función de bienestar social en que se determinan los objetivos a perseguir por el Estado y sus jerarquías, de manera generalmente implícita. También el análisis político clarifica la selección de megapolíticas, al facilitar la compresión de los problemas de poder y negociación (Cardozo, 1990, p. 183).

La estrategia combativa de vincular elementos como causas de la situación de crisis - como en el caso de la migración — ha debilitado la capacidad de diálogo internacional y la posibilidad de generar propuestas dirigidas a restablecer el orden en diferentes frentes. Ahora la emergencia social se traslada de los países europeos o norteamericanos a los emergentes, pues, además de divisas y recursos, habían depositado en ellos las perspectiva de mejorar las condiciones de vida de quienes, animados por unas mejores oportunidades, habían decidido emigrar, crear empresas o ubicar recursos de diferentes orígenes en países de Latinoamérica, donde las políticas eran salvaguardia no de un modelo económico, sino del interés por el bienestar mundial en general. En este sentido, Elizalde (2003) señala como ventajas que se persiguen en estos territorios con la implementación de las políticas de desarrollo local: "Mejorar la calidad de vida y bienestar social de los ciudadanos; reducción de la dependencia exterior; reforzamiento del espíritu colectivo; crecimiento y generación de empleo; conservación del medio natural; desarrollo cultural de la comunidad" (p. 40).

Según lo anterior, es el momento para que los países emergentes replanteen los modelos que pueden seguir, y que se entienda que ellos 
requieren compromiso o sacrificio para estar a la par con los países desarrollados, que han mostrado que el diseño de sus modelos también está expuestos a desbarajustes, que no necesariamente tienen arreglo, y que si lo tienen, es a cuesta precisamente de lo que, en medio del optimismo, decidieron honrar por algún tiempo, pero que hoy constituye su base de deterioro a escala internacional. En este sentido, cabe apreciar las bondades de contar con políticas públicas internacionales coherentes con políticas estatales audaces y con un alto impacto en su implementación; políticas con real asidero y asiento, para poder aplicarlas completamente:

El papel del Estado es central en una crisis de dimensión mundial. Dada la extrema fragilidad de los instrumentos de gobierno global, el eje estratégico de construcción de los nuevos sistemas de regulación pasará más por la articulación de políticas nacionales que por los avances en una esfera propiamente global. El Estado aparece, entonces, con una función reforzada, tanto en lo que respecta a los equilibrios internos como en la redefinición de las reglas del juego entre las naciones (Dowbor, 2009, p. 189).

Las políticas públicas han pasado de ser herramienta útil a constituir la columna y base del diseño de propuestas para revertir las circunstancias difíciles en las que se encuentra la base estatal en los países latinos. Las políticas derivadas de un sometimiento ciego a planes y programas establecidos desde organismos multilaterales han reducido el papel del Estado, lo han convertido en asimilador de recetas, pero sin ninguna garantía de que funcionen; no obstante, forman parte del diálogo de obligaciones que deben ejecutar estos países para no estar fuera de los preceptos de un modelo mecánico que tiene a todo el mundo al borde del colapso, y a los Estados en la obligación de redefinir su papel en las circunstancias derivadas de la evidente crisis:
La restauración del Estado, antes que su pretendida reforma o modernización, requiere de una revalorización de lo público. La restauración de lo público supone cuando menos la instalación de lo público por tres vías: la política democrática, la re-territorialización de la vida social y la formación de mecanismos no mercantilizados de movilidad social, es decir, bienes públicos para el desarrollo (Sojo, 2005, p. 152).

La política pública enfrenta, como pocas áreas de aplicación, una serie de altibajos relacionados con los elementos que la apropian para destacar las acciones que, desde distintos frentes, realizan quienes comprenden la relevancia de poner en acento el desarrollo, el ordenamiento o la planeación. A su vez, muchos otros la utilizan como mecanismo para develar, a manera de diagnóstico, las condiciones que se presentan en dichos acentos o estrategias, por cuenta igualmente de entidades ejecutoras o evaluadoras de los resultados en la gestión pública.

\section{Estructura compleja y sistémica de la política pública ante la crisis internacional en los países emergentes latinoamericanos}

El campo ocupado por los países emergentes, otrora denominados en vías de desarrollo, ha formado parte del papel cumplido por estos respecto a los países desarrollados o industrializados. En orden de relevancia, la significancia de las políticas internacionales ha considerado el orden que cada país latinoamericano ocupa a la hora de incentivar o promover alguna tarea conjunta bilateral o multilateral determinada por los organismos internacionales. El objetivo de ello es orientar las diferentes políticas que, en calidad de vinculantes, tienen asiento en los países, en un mundo en el que la bipolaridad regía el orden internacional y luego pasaría a afianzarse de manera unilateral. Sin embargo, ahora el orden mundial ha desaparecido. 
La ausencia de países líderes en el orden internacional demuestra una de las tantas consideraciones desde las que se sustenta la idea relacionada con el aprovechamiento del marco de diferencias y de sacar provecho de las oportunidades que, aun con reparo, deberían ofrecer los países. Ahora, bajo el precepto de la homogeneidad, la incertidumbre - que antes era propia del estado de guerra permanente de la época bipolar - ha pasado a caracterizar el orden internacional. Este suceso no encuentra explicación lógica para establecer el origen de tal desfallecimiento global, sobre el cual ni el Estado ni cualquier otra institución que pretenda procurar un orden encuentran receptividad y trascendencia particular en los países de Latinoamérica. Giménez (2011) sostiene al respecto:

Se configuró así un Estado cuya pérdida progresiva de sus capacidades lo dejó muy vulnerable a las crisis, con pocas posibilidades de revertirlas o de evitarlas. $Y$ esto colocó al fundamento del Estado y lo público en cuestión como nunca antes. El Estado y lo público quedaron así en la picota, en el centro de los cuestionamientos de la sociedad y sin capacidad de reaccionar por el recorte de las atribuciones y poderes estatales que concretó el neoliberalismo. El Estado no garantizó la propiedad, no brindó seguridad y dejó de ser reconocido como institución por negársele el derecho de representación a la clase política. En síntesis, quedó reducido a ejercer el poder coercitivo, represivo.

El paso creciente de los cuestionamientos hechos al modelo derivado del capitalismo y las pretensiones que tras de el eran estimadas, emprenderían el impulso para que a medida que se ejecutaban las políticas estatales, estas fueran evaluadas en cuanto a los resultados y transformaciones que de manera consecuente se esperaban. Puede decirse que este primer llamado de atención permitió la vigencia de los fundamentos que enmarcarían el campo de acción de las políticas públicas. El alcance, los impactos y logros pasarían a ser el punto de llegada al de partida para establecer las bases de dirección y fundamentación de la política pública como principio de recambio a la trayectoria definida por el campo de las políticas estatales (p. 78).

La preocupación que motivó el interés por establecer unos lazos claros en materia de política pública corrió por cuenta de los mismos actores institucionales de diversos países latinoamericanos, que acusaban la falta de claridad y asiento de los programas o proyectos que llevaban a cabo, por cuanto no se cumplían los objetivos; a la par, terminan por profundizar aún más las circunstancias y razones que les habían dado origen. El propósito de evidenciar claramente el alcance y los logros de los planes y programas estatales y multilaterales permitiría diseñar otra serie de propuestas metodológicas para perfilar la política estatal como marco público de acción, entre las que se destacan el marco lógico y el análisis sistémico.

El marco lógico apunta a que los objetivos planteados en los programas y proyectos, además de conseguirse, sirvan de puente para la participación de la población en lo concerniente a la elaboración, el diseño, la evaluación y la orientación que, por claras circunstancias, deberían contar con la intervención estatal en cualquier espacio o sobre algún tema en especial. En esa misma dirección se orienta el análisis sistémico, pero en un esquema ordenado: una vez acoplada la fundamentación del marco lógico, pasa a entenderse como un sistema cuyos procesos se comportan de manera abierta y cerrada, para que sean reprocesados o permitan la interacción de diversos componentes que pueden mejorarla.

A modo de avance, se plantea como marco de discusión respecto a los anteriores fundamentos esquemáticos de evaluación algunos elementos concernientes al análisis complejo. Esto, de manera abierta y propositiva, pretende contribuir a estimar 
las bases de las políticas o de cualquier otro campo, sin más obstáculos que la necesidad de entender el entorno y el panorama internacional, así como los elementos cruzados y las variables que lo constituyen. En correspondencia con las circunstancias, cualquier elemento puede ser relevante o importante para redireccionar los fundamentos que pueden estar fallando de manera individual, pero que en concreto pueden estar siendo atomizados o sustentados por otros elementos no considerados.

Los análisis de estructuras complejas, de marco lógico y sistémico perfilan el alcance y las bases de las políticas públicas, y no solo como ejercicio para la aplicación directa, sino como fundamento de forma y fondo que se requiere para que estas tengan asiento en el resto de estructura: el escenario de trabajo de las políticas estatales latinoamericanas. Por encima de su procedencia, estas últimas tienen aforo en las instancias regionales y territoriales, donde se reclama un puente de mayor acción ya sea por parte del Estado o de instituciones que, desde distintas vertientes, traen consigo programas y proyectos de orden estatal, regional o local.

\section{Crisis internacional y estados de emergencia entre los países Latinoamericanos desde las políticas públicas}

La conmoción provocada por el estancamiento de la economía mundial y el auge de políticas restrictivas presentadas caracteriza las relaciones internacionales entre los países. En su defecto, los países desarrollados han decidido tomar por su cuenta las riendas de la situación, ajenas a las instituciones multilaterales creadas precisamente para contribuir o prevenir para que estas circunstancias tuvieran alguna posibilidad de superación. El grado de restricciones ha llegado a tal algidez que han sido restablecidas políticas antinmigrantes, implementación de políticas de ajuste fiscal, al comercio y las inversiones internacionales.
El alcance de las políticas acogidas por los países desarrollados ha terminado por incidir en las bases económicas de los países emergentes, que observan, impávidos, el proceso que está tomando la crisis, pero que de manera poco contundente han afinado los fundamentos económicos, legales y políticos para que el colapso que pueda traer la crisis no sea más profundo. De hecho, la vulnerabilidad ha crecido por cuenta del aumento de los vínculos entre naciones. Las políticas de ajuste tomadas en los países emergentes no han pasado por una extensiva revisión; la consecuencia puede ser más grave de lo esperado:

El desarrollo económico de los distintos países sigue siendo territorialmente concentrado e inequitativo, razón por la cual no ha sido posible aprovechar el potencial productivo de los distintos territorios, lo que a su vez constituye una de las causas de aumento de la pobreza. Por consiguiente, el análisis del papel que tienen las localidades en la promoción del desarrollo económico dentro de un contexto de gestión descentralizada está en la agenda de los temas emergentes para los países de la región (Lira, 2003, p.11).

En consecuencia, de las políticas de choque tomadas por los países desarrollados y de la postura de gravidez adoptada por los emergentes se derivará la situación de la población natural o nacionalizada, pero también de la ilegal e inmigrante, que no tiene certeza de las condiciones en que se encuentra el país donde reside ni mucho menos de lo que puede pasar en el frente internacional. La debilidad del dólar y del euro contrasta con la fortaleza de los medios de pago de los países emergentes, contexto en el que los mecanismos de intercambio han puesto en jaque la sostenibilidad de los flujos económicos internacionales. La discusión se dirige precisamente a confrontar los de la economía mundial. La carrera por contar con un alto grado de homogeneidad y reciprocidad de 
los países ha pasado a representar una disparidad lógica, que es infranqueable entre los países y que solo es visible cuando entre ellos, aun unidos, no se reflejan propuestas claras para revertir el desajuste o las consecuencias provocadas por la crisis. Ahora el turno corre por cuenta del ajuste de las cuentas macroeconómicas, desde las que se evalúa hasta dónde pueden llegar los países de seguir la crisis en dicha senda:

La pérdida de credibilidad del sector público como gestor del bienestar ciudadano viene acompañado de manifestaciones que exigen y obligan a que las instituciones públicas actúen aplicando principios de economía, eficiencia y eficacia. Estas nuevas demandas, cada vez más importantes como consecuencia de la magnitud del déficit público en prácticamente la totalidad de países, tuvieron en un primer momento un importante impacto en los procesos de construcción de políticas públicas, pero en la actualidad no se circunscriben únicamente a la esfera política o ministerial, sino que se han ampliado a todas las actuaciones de la administración en sus diferentes órdenes territoriales (García, 2007, p. 38).

Los llamados a las restricciones forman parte de los fundamentos de política económica adoptados por los países emergentes, a diferencia del imperativo categórico que con respecto a ellos debe hacerse en los países desarrollados. Unos y otros han adoptado decisiones paralelas sobre cómo afrontar la crisis; aun así, han tomado de forma unívoca la idea de dejar a un lado las bondades de las políticas públicas para disminuir el impacto de la crisis, de los ajustes que se encuentran realizando todos los países latinoamericanos y los choques que entre ellos provoca la toma de decisiones unilaterales establecidas:

De acuerdo con la perspectiva analítica aquí asumida, consideraremos cinco grandes estrategias de política pública que constituyen los pilares de la reforma del Estado en nuestro continente en lo que se refiere específicamente a las transformaciones que se han pretendido implantar con el fin de modificar la naturaleza y funcionalidad de las burocracias público-estatales. Son ellas: 1) el desmonte del Estado empresario o empresarial; 2) la promoción de la competencia y la liberalización de la política económica y social; 3) las reformas políticas propiamente dichas, de las cuales destacaré principalmente las laborales, incluyendo cuestiones vitales en el campo de la seguridad social; 4) las políticas de descentralización, en especial las de transferencia de competencias, responsabilidades $y$ recursos desde el nivel central del Estado, a las regiones y las localidades; $y$, en último lugar, 5) el emprendimiento de numerosas transformaciones organizacionales de la estructura y la funcionalidad del Estado, las cuales se han dirigido a atacar el corazón de la burocracia, pretendiendo transformar los mecanismos de gestión, diseños, organizaciones, formas de control, y evaluación del desempeño, modalidades de asignación presupuestal, etc. (Varela, 2005, p. 259).

El primer resultado que arroja la crisis es el estado de desolación con que se encuentran cada uno de los países en Latinoamérica para revertir las consecuencias de lo sucedido. Es evidente que la estructura multilateral creada por los países no opera; prueba de ello es la ausencia de los principales organismos multilaterales para dar la cara a algo que forma parte de sus tareas, como es la función de evaluar la dirección tomada por la economía mundial y ofrecer, con cargo a ello, las recomendaciones a las que hubiese lugar de manera diferencial para cada país, conforme al rango de impacto. La disparidad de circunstancias es la que extiende la aparición de las políticas públicas. 
A este respecto, Babino (2009) sostiene:

La ausencia de una visión sistémica en los procesos modernizadores o reformadores del Estado dio lugar a un proceso de reforma de los "sistemas horizontales" de gestión, como también sobre las instituciones de forma "vertical". La teoría de sistemas postula que el sistema general opera en el nivel de calidad del menor de los subsistemas que lo integran. Por esto cuando se encaran procesos de transformación, es preciso prestar atención tanto a las mejoras de sistema como a las interrelaciones que se dan entre ellos (p. 6).

La madurez de las políticas públicas corre por cuenta de la comparación del desarrollo institucional que han tenido los territorios en el interior de los países latinoamericanos. En consecuencia, el estado de emergencia, colmado por las circunstancias y la complejidad, ha mostrado en los países emergentes que el estado de crisis es un hecho crónico de su realidad; por ende, el asomo o la reaparición de circunstancias como estas solo sirven para apreciar la dispersión y la tendencia de las variables en las gráficas de los magazines económicos, pero nada que advierta un escenario sombrío o sorpresa:

El valor del enfoque de las políticas públicas radica en su preocupación por la acción del gobierno y sus administraciones. No se interesa tanto por la estructura y composición del sistema político-administrativo como por su funcionamiento. El análisis de políticas es a la vez un arte y una ciencia. Es un arte porque hace referencia a la labor de los directivos públicos, que intuitivamente reproducen con sus decisiones las fases del proceso de las políticas sin dominarlo conceptualmente. Es una ciencia porque aspira a entender el complejo mundo de la acción pública y prescribir, en la medida de lo posible, la mejor forma de aproximarse al proceso de las políticas (Tamayo, 1997, p. 27).

La regularidad que han alcanzado las políticas públicas para mediar con las políticas económicas emprendidas en los países emergentes latinoamericanos desdice de la distancia tomada por ellas con respecto a los países desarrollados. Diversas comprensiones pueden sumarse a la inexplicable dilatación de la política pública en las regiones de los países emergentes en comparación con los desarrollados. Los países emergentes hoy recaban en sus políticas estatales e institucionales para resolver un problema que tiene asiento en la capacidad de cada territorio para avanzar en las circunstancias, con el acompañamiento estatal:

Las políticas públicas responden a necesidades complejas que no tienen un origen específico y representan dinámicas que no tienen causales específicas o dinámicas claras. Así, al pretender que este tipo de políticas solucione de manera efectiva anomalías complejas que no tienen una causa particular, a su vez, generan otras problemáticas. Hay que tener claro que la política debe ser integral y articulada con otras políticas para poder garantizar una atención global a la problemática y a sus consecuencias. Pero si, por el contrario, la política tiene una orientación sectorial, difícilmente los problemas serán solucionados (Arroyave, 2010, p. 108).

La posición multilateral adoptada por los países desarrollados para repeler la crisis es comparable con la perspectiva central y territorial tomada por los emergentes para procurar mejorar los fundamentos macroeconómicos, con impacto en las regiones que de manera diferencial deben sopesar los enviones que las crisis provocan. De allí que, a diferencia de los países europeos, la política estatal en los territorios de los países emergentes se basa en toda la fundamentación de la política pública, caracterizada por ubicar a la población en el centro de interés y foco de resultados con las políticas 
y los recursos disponibles. Tamayo (1997) es cercano a esta aproximación:

El concepto de política pública es inseparable de la noción de análisis de políticas; la política pública no existe en la realidad, sólo la intervención del analista otorga un sentido a la multitud de decisiones $y$ comportamientos de los administradores y de los demás actores sociales involucrados en el proceso. La riqueza del análisis radica en su capacidad para integrar y ordenar lógicamente esas decisiones y comportamientos. El análisis de políticas públicas es un campo de especialización profesional emergente que está cobrando una gran importancia. El análisis, la evaluación, la investigación aplicada al proceso de formación de las políticas no es un lujo, es una necesidad que habrán de afrontar las organizaciones públicas para asegurar su supervivencia. Sólo mediante políticas eficaces - desde el punto de vista del impacto social que generan y de la eficiencia en la utilización de los recursos - se puede legitimar, efectivamente, la acción de los gobiernos en una democracia pluralista (p. 2).

La crisis internacional que hoy rodea a los países desarrollados constituye en sí la reproducción acumulada de las crisis que se han venido presentando desde comienzos de los ochenta (década perdida para los países de América Latina) y con las circunstancias presentadas en los noventa (crisis en los países asiáticos) y comienzos del siglo XXI (para el caso de los países europeos y Estados Unidos). En otras palabras, la cuenta de cobro de la crisis se ha presentado en la mayoría de países de Occidente; situación estructural que ha terminado por deteriorar la capacidad de respuesta individual de los países.

Las cumbres convocadas para definir las estrategias y fórmulas para revertir las consecuencias de la actual crisis han partido de la idea de procurar mecanismos restrictivos de diversa índole. Ha sido tan poca la capacidad y el impacto de las medidas tomadas para contrarrestar las consecuencias de las crisis que mecanismos considerados discriminatorios forman parte de las variables que se pretenden imponer para mejorar las condiciones de los países embarcados particularmente en una situación que ha empeorado hasta la fecha actual; es el caso de las políticas antimigratorias, el cierre de fronteras económicas, el control de cambios o la restricción al uso de divisas.

La tabla 1, a modo de comparación, referencia países latinoamericanos como estados y territorios emergentes: Argentina, Brasil, Chile, Colombia y México, en el periodo 2003-2007, en el marco de las políticas públicas para afrontar crisis económicas y sociales. Estas crisis de naturaleza financiera, de legitimidad y eficacia en los sistemas y de garantía de seguridad y protección social están relacionadas proporcionalmente con el crecimiento económico para mantener la prestación constante de servicios y bienes sociales; sin embargo, los factores políticos y las políticas gubernamentales que originan la expansión y consolidación del bienestar con base en el financiamiento público condicionan su mantenimiento. De ahí que la adopción de políticas públicas posibilite medidas de aseguramiento que permitan contrarrestar las crisis económicas y sociales, a través de agendas públicas a escala de gobiernos subnacionales y de la asignación y priorización de recursos orientados a resultados.

Las crisis económicas y sociales en estos Estados y territorios emergentes están focalizadas, por un parte, en la institucionalidad, por la alta burocratización para el cumplimiento de los fines del Estado latinoamericano y la crisis de legitimidad institucional, que está fuertemente incidida por la corrupción y la falta de coordinación interinstitucional con los gobiernos subnacionales; y, por otra parte, en los procesos presupuestarios, debido a la constante necesidad de financiamiento del bienestar social.

Por ende, los esfuerzos de las políticas públicas adoptadas por estos países ante las crisis económicas y sociales se encaminan en varios frentes: a) sostenimiento de las fuentes generadoras de empleo; b) desarrollo de reformas tributarias (carga impositiva); c) estimulación de la producción 
Tabla 1.

Esquema de las políticas implementadas como respuesta a la crisis internacional

\begin{tabular}{|c|c|c|c|c|c|}
\hline \multirow[b]{2}{*}{ Medidas } & \multicolumn{5}{|c|}{ País } \\
\hline & Argentina & Brasil & Chile & Colombia & México \\
\hline \multicolumn{6}{|c|}{ Política monetaria y financiera } \\
\hline Disminución y/o flexibilización del encaje bancario & $\mathrm{x}$ & $\mathrm{x}$ & $x$ & $\mathrm{x}$ & \\
\hline Provisión de liquidez en moneda nacional & $\mathrm{x}$ & $\mathrm{x}$ & $\mathrm{x}$ & $\mathrm{x}$ & $\mathrm{x}$ \\
\hline \multicolumn{6}{|c|}{ Política fiscal } \\
\hline Disminución de impuestos/aumento de subsidios & $\mathrm{x}$ & $\mathrm{x}$ & $\mathrm{x}$ & $\mathrm{x}$ & $\mathrm{x}$ \\
\hline Aumento y/o anticipación del gasto (infraestructura) & $\mathrm{x}$ & $\mathrm{x}$ & $x$ & $\mathrm{x}$ & $\mathrm{x}$ \\
\hline \multicolumn{6}{|c|}{ Política cambiaria y de comercio exterior } \\
\hline Provisión de liquidez en moneda extranjera $\left({ }^{*}\right)$ & $x$ & $x$ & $x$ & $\mathrm{x}$ & $\mathrm{x}$ \\
\hline Aumento de aranceles o restricciones a las importaciones & $\mathrm{x}$ & & & & \\
\hline Disminución de aranceles & & $\mathrm{x}$ & & & $\mathrm{x}$ \\
\hline Financiación a exportadores & $\mathrm{x}$ & $\mathrm{x}$ & $\mathrm{x}$ & $\mathrm{x}$ & \\
\hline Financiación multilateral & $\mathrm{x}$ & & $\mathrm{x}$ & $\mathrm{x}$ & $\mathrm{x}$ \\
\hline \multicolumn{6}{|c|}{ Políticas sectoriales } \\
\hline Vivienda & $x$ & $\mathrm{x}$ & $x$ & $\mathrm{x}$ & $\mathrm{x}$ \\
\hline Pymes & $\mathrm{x}$ & $\mathrm{x}$ & $\mathrm{x}$ & $\mathrm{x}$ & $\mathrm{x}$ \\
\hline Agropecuario & $\mathrm{x}$ & $\mathrm{x}$ & $\mathrm{x}$ & & $\mathrm{x}$ \\
\hline Turismo & & & & & $\mathrm{x}$ \\
\hline Industria & $\mathrm{x}$ & $\mathrm{x}$ & $x$ & $x$ & $x$ \\
\hline \multicolumn{6}{|c|}{ Política laboral y social } \\
\hline Estímulo al empleo & $x$ & $\mathrm{x}$ & $x$ & $x$ & $x$ \\
\hline Programas sociales & $x$ & $\mathrm{x}$ & $x$ & $x$ & $x$ \\
\hline
\end{tabular}

${ }^{*}$ ) No incluye a los bancos centrales que intervinieron vendiendo divisas en los mercados de cambio. Fuente: Ronconi, Marongiu, Dborkin y Filc (2010, p. 18).

industrial y agropecuaria, a través de créditos con tasas moderadas, acciones económicas y fiscales a largo plazo; d) disciplina fiscal en temas de déficit y gasto público, y e) infraestructura social y económica-productiva; la primera, enfocada a mejorar las condiciones habitacionales, como vivienda, agua, riego, drenaje y saneamiento; la segunda, orientada a comunicación y transporte (mantenimiento y extensión de la red vial, puentes, conexiones viales, puertos, ferrocarriles, transporte urbano e interurbano, aeropuertos), energía (generación y transporte de energía eléctrica y renovable 
y minería) y medioambiente (gestión y conservación de recursos naturales).

Según Mascareño (2003), en los estados emergentes las políticas públicas para afrontar las crisis económicas y sociales deben ser transversales, en cuanto a objetivos y resultados:

a. Las políticas de redistribución, cuya base principal es la política fiscal, tienden a ser más aisladas de las fuerzas externas globales, pues las mismas se encuentran altamente condicionadas por factores domésticos de acuerdo con la base legal interna y la propia evolución histórica que las constriñe a los ámbitos locales.

b. Las políticas regulativas, por el contrario, van adquiriendo un perfil altamente global y, dentro de él, uno regionalizado. Trátese de medio ambiente, migraciones, drogas, armas nucleares, terrorismo o comercio, las soberanías tienen que ceder ante los intereses múltiples de bloques de países y aun de todos los países.

c. Las referidas a la modernización y la democracia aluden directamente a la ampliación creciente de los derechos, con lo cual la presencia de actores extraterritoriales, supraestatales y no gubernamentales, es insoslayable. La presión por los derechos humanos, las reformas de los sistemas de justicia, la transparencia de los sistemas electorales o la rendición de cuentas públicas son políticas que atraviesan a naciones y partidos políticos.

d. Finalmente, con un creciente carácter global, se ubican las políticas de liberalización. Éstas, caracterizadas por la desregulación de los mercados, la difusión de tecnología y la integración financiera en mercados de capitales, atraviesan las naciones y superan las posibilidades de las fronteras soberanas. En particular, estas últimas han introducido altos niveles de ingobernabilidad en el sistema financiero global.

Así, los servicios sociales que profieren las políticas públicas en los países latinoamericanos están en relación con la financiación y el tamaño del sector público, como el garante de la cobertura y eficiencia en la provisión de bienes y servicios. Sin embargo, en Argentina, Brasil, Chile, Colombia y México se hizo necesario sectorizarlas para mayor intervención del Estado, como articulador de la inserción social. Esto implica gestión de gobierno subnacional y transnacional y complejizar las realidades en asuntos como asentamientos urbanos, asuntos públicos en equidad, género, derechos humanos, dinámicas demográficas y agroindustria.

Además, dadas las particularidades de los territorios subnacionales, asociados a la vida política, económica y cultural local, deben existir particularidades en el tratamiento de una política pública nacional, para la constante necesidad de financiamiento del bienestar social. Los Estados y territorios emergentes como los países latinoamericanos deben profundizar su papel estatal en cuanto a regular los intereses individuales y colectivos que intervienen en el desarrollo de la política pública. Por su parte, el ciudadano y la sociedad deben articularse a la gestión de asuntos públicos, en función de superar las tradicionales políticas sectoriales, con una política pública cohesionadora y una acción contracíclica ante las crisis económicas y sociales:

Un elemento interesante que también repercute en la forma en que los países encaran acciones contra-cíclicas es la existencia o no de Planes Nacionales de Desarrollo (presentes en Brasil, Colombia y México). A grandes rasgos, puede concluirse que estos instrumentos no sólo facilitan la respuesta de política al permitir contar con prioridades ya definidas, sino 
que también abren la puerta a una mayor consistencia de la respuesta con la estrategia de largo plazo (aunque no garantizan el éxito en su implementación). Si bien la simple existencia de estos instrumentos no implica que las políticas adoptadas en tiempos de crisis se adecuen a ellos, en mayor o menor medida los países estudiados se han apoyado en esas herramientas. Por ejemplo, como se observó anteriormente, tanto México como Colombia centraron sus esfuerzos en la ampliación del gasto en infraestructura, con medidas generalmente vinculadas a las prioridades preestablecidas. En el caso de Brasil, los sectores beneficiados por las medidas sectoriales se vinculan a la estrategia de desarrollo industrial preexistente plasmada tanto en la Política Industrial, Tecnológica y de Comercio Exterior (PITCE) como en el Plan de Desarrollo Productivo. De todos modos, también se encuentran ejemplos en donde la urgencia por estimular la economía y por atender las necesidades que surgieron con la crisis impulsaron que se destinen recursos a sectores o actividades que no habían sido contemplados como prioritarios en la estrategia de desarrollo de largo plazo (Ronconi et al., 2010, p. 85).

De esta manera, las políticas públicas, desde la perspectiva de las crisis económicas y sociales, en el marco de los Estados y territorios emergentes latinoamericanos, se traducen en nuevas formas de gobernabilidad, donde los asuntos públicos son interdependencias de continuo proceso en el que hay funcionamiento de relaciones de poder e intereses sociales, una institucionalidad centralizada en cuanto a responsabilidades y autoridad a los gobiernos subnacionales y la eliminación de la burocracia inoperante. En otras palabras, son unos Estados y territorios emergentes con características de este tipo: gobiernos competitivos en la prestación de servicios y bienes públicos; gobiernos dirigidos a resultados en materia en la satisfacción de las necesidades básicas, la sostenibilidad económica y fiscal; y gobiernos enfocados en el ciudadano como cliente, es decir, satisfacer más demandas para que la estructura burocrática, a su vez, controle y priorice su gasto público.

\section{CONCLUSIONES}

El modelo actual de políticas públicas no ha tenido el acierto esperado en materia de condiciones que permitan orientar y soportar los altibajos presentados en la economía y la política internacional. La enseñanza en este sentido responde a la capacidad real que tienen los países latinoamericanos para contribuir articuladamente a resolver dificultades que no son estimadas en medio del apogeo o que, por cuenta de salvaguardar procesos legales y políticas, pueden evitarse o prevenirse. En este sentido, la actual crisis indica que las políticas estatales deben estimar en congruencia paralela a las políticas públicas.

Además, el contexto del desarrollo de las políticas públicas está suscrito a las sociedades democráticas, a través de valores administrativos como la eficacia y eficiencia, y de valores democráticos como la transparencia, equidad, responsabilidad pública y defensa de derechos. De esta manera, sería posible ampliar el marco de legitimidad y preservación del sistema político-administrativo latinoamericano, a través de la garantía en el acceso y financiamiento, para ser reinvertidos en los planos administrativos y sociales. La señal presentada por los países desarrollados (europeos y Estados Unidos) muestran una clara debilidad estatal e institucional para solucionar problemas que surgieron precisamente por no contar con elementos concisos a la hora de defender los avances del modelo económico reinante o las políticas internacionales ejecutadas bajo la consigna de recetas, pero que hoy, como prueba de su inoperancia, han arrastrado consigo a los países hacedores. $Y$ de no detenerse rápidamente, tendrán consecuencias notorias en los países discrecionalmente llamados emergentes, como las naciones latinoamericanas. 
Un elemento que se aproxima al patrón de vaguedad que sirve para llamar como tal a las políticas públicas es cuando estas son tomadas para reflejar el vacío institucional o la ausencia de la administración pública con respecto a sus funciones y quehaceres. Esta es una percepción un tanto antagónica y fuera de tono de la capacidad que tienen las políticas para hacerse sentir por encima de las posibles elucubraciones que podrían darles origen. Es claro que para los Estados latinoamericanos y sus instituciones es importante cultivar un marco de entendimiento con los ciudadanos, y no hay terreno más expedito para lograrlo que con la mediación de las políticas públicas.

\section{REFERENCIAS}

1. Aguilar, L. (2007). El aporte de la política pública y de la nueva gestión pública a la gobernanza. Reforma y Democracia, 39, 1-15.

2. Arroyave, S. (2010). Las políticas públicas en Colombia. Insuficiencias y desafíos. Forum, 1, 95-111.

3. Babino, L. (2009). Hacia una visión sistémica de la gestión de gobierno. XV Congreso Internacional del CLAD sobre la Reforma del Estado y de la Administración Pública. Buenos Aires: Centro Latinoamericano de Administración para el Desarrollo.

4. Bayce, R. (2005). Políticas públicas: bienestar, reconocimiento, simulacro. Ciências Sociais Unisinos, 44(1), 69-79.

5. Cardozo, M. (1990). Caracterización de las técnicas de evaluación de las acciones de organización públicas. En E. Ortiz (comp.), Administración pública, economía y finanzas: países actuales en los países de Norteamérica y el Caribe (pp.167-197). Ciudad de México: Centro de Investigación y Docencia Económicas.

6. Dowbor, L. (2009). La crisis no es solo económica. Reflexiones globales y algunos apuntes sobre Brasil. Revista Nueva Sociedad, 224, 179-199.

7. Elizalde, A. (2003). Planificación estratégica territorial y políticas públicas para el desarrollo local. Santiago de Chile: Cepal.

8. $\quad$ Esser, K., Hillebrand, W., Messner, D., y Meyer-Stamer, J. (1994). Competitividad sistémica. Competitividad internacional de las empresas y políticas requeridas. Berlín: Instituto Alemán de Desarrollo.

9. Feoli, L. (2011). Instituciones políticas y políticas públicas ante la crisis global. Nueva Orleans: Universidad Tulane.

10. García, I. (2007). La nueva gestión pública: evolución y tendencias. Salamanca: Universidad de Salamanca.

11. Giménez, S. (2011). Lo público estatal, la exclusión y las políticas sociales. Neoliberalismos y después. Debate Público, 1, 75-85.

12. Lahera, E. (2004). Política y políticas públicas. Santiago de Chile: Cepal.

13. Landes, D. (1998). The wealth and poverty of nations: why some are so rich and some so poor. Nueva York: W.W. Norton.

14. Lira, L. (2003). La cuestión regional y local en América Latina. Santiago de Chile: ILPES- Cepal.

15. Manuel, A. (2005). La intervención en lo social, las problemáticas sociales complejas y las políticas públicas. Margen, 39, 1-9.

16. Mendíaz, M. (2004). El Estado y las políticas públicas. Las visiones desde el neoinstitucionalismo. Rosario: Universidad Nacional de Rosario. 
17. Mascareño, C. (2003). Las políticas públicas ante las realidades emergentes. Cuadernos del Cendes, 52. Recuperado de http://www.scielo.org.ve/scielo.php?script $=$ sci_arttext\&pid $=$ S1012-25082003000100003

18. Ronconi, L., Marongiu, F., Dborkin, D. y Filc, G. (2010). América Latina frente a la crisis internacional: características institucionales y respuestas de política. Madrid: Fundación Carolina.

19. Sojo, C. (2005). La modernización sin Estado: el destino privado de las políticas públicas. En Facultad Latinoamericana de Ciencias Sociales (Flacso), La gobernabilidad en América Latina. Balance reciente y tendencias a futuro (pp. 137-154). San José de Costa Rica: Flacso.

20. Tamayo, M. (1997). El análisis de las políticas públicas. En R. Bañon y E. Carrillo (comps.), La nueva administración pública (pp. 1-22). Madrid: Universidad Complutense de Madrid e Instituto Universitario Ortega y Gasset.

21. Varela, E. (2005). La transformación de la burocracia pública: una perspectiva crítica del proceso de reforma del Estado en América Latina. En La mercantilización de lo público: ensayos sobre gestión y políticas públicas (pp. 255-27). Cali: Universidad del Valle. 